\title{
Exotic massive gravity: Causality and a Birkhoff-like theorem
}

\author{
Ercan Kilicarslan ${ }^{1, *}$ and Bayram Tekin ${ }^{2, \dagger}$ \\ ${ }^{1}$ Department of Physics, Usak University, 64200, Usak, Turkey \\ ${ }^{2}$ Department of Physics, Middle East Technical University, 06800, Ankara, Turkey
}

(Received 26 June 2019; published 16 August 2019)

\begin{abstract}
We study the local causality issue via the Shapiro time-delay computations in the on-shell consistent exotic massive gravity in three dimensions. The theory shows time delay as opposed to time advance despite having a ghost at the linearized level both for asymptotically flat and anti-de Sitter spacetimes. We also prove a Birkhoff-like theorem: any solution with a hypersurface orthogonal non-null Killing vector field is conformally flat; and we find some exact solutions.
\end{abstract}

DOI: 10.1103/PhysRevD.100.044035

\section{INTRODUCTION}

Three-dimensional spacetime is poor in massless gravitons, yet very rich in massive ones: in addition to the well-known topologically massive gravity (TMG) [1], new massive gravity (NMG) [2], cubic [3], and Born-Infeld extensions [4], which are all based on actions that depend on the metric alone, a new set of theories that lack a purely metric-based action have been found in [5-7]. The field equations of these theories are on-shell consistent; namely, they possess a Bianchi identity for the metrics that solve the field equations but not for generic off-shell metrics. These theories are highly restricted [8,9]. Here we are interested in the more recent theory, the so-called "exotic massive gravity" (EMG) defined in [10] and extended and elaborated in various aspects [11-14]. One of the main reasons to search for new theories in $2+1$ dimensions is to try to construct a bulk and boundary unitary theory which would amount to defining a quantum theory of gravity via the AdS/ CFT conjecture. See the summary of the unitarity problem in three-dimensional massive gravity theories in [15].

Following the discussion of causality of extended gravity theories in $[16,17]$ using the Shapiro time-delay computation [18], we study the causality of EMG both in asymptotically flat and anti-de Sitter (AdS) spacetimes. It was realized in [16] that the Einstein-Gauss-Bonnet theory is not causal even in the regime when the theory is unitary and moreover the addition of finite number of curvature terms in the theory does not solve the problem. The problem has a

\footnotetext{
*ercan.kilicarslan@usak.edu.tr

†btekin@metu.edu.tr
}

Published by the American Physical Society under the terms of the Creative Commons Attribution 4.0 International license. Further distribution of this work must maintain attribution to the author(s) and the published article's title, journal citation, and DOI. Funded by SCOAP. solution in string theory $[16,19]$ with an infinite tower of massive intermediate states. This naturally prompted the question as to whether three-dimensional theories suffer from causality violation. It was shown in [17] that, unlike the higher dimensional theories, causality does not bring in new constraints beyond the unitarity constraints in the thenknown three-dimensional massive gravity theories. Since that work, the EMG theory has emerged and a similar computation in this theory is one of the tasks of this work.

As discussed in [17], the usual computation of the Shapiro time delay of a signal is done for a round trip in a black hole background for more than three spacetime dimensions. However, in three dimensions, using the motion of test particles or fields in a shockwave geometry $[20,21]$ created by a massless particle is better suited. This is because we do not know black hole solutions in these theories other than the Banados-Teitelboim-Zanelli black hole [22] which is only obtained after identifying points of $\mathrm{AdS}_{3}$; and therefore it is not suitable for local causality discussions via the time-delay arguments. Here we show that even though EMG is known to be ghost-ridden at the linearized level, it nevertheless yields a time delay, instead of an advance, for fields and test particles, which is consistent with causality.

In addition to the causality discussion, we also prove a theorem which is in some sense analogous to the Birkhoff theorem in four dimensions: all solutions of EMG that possess a hypersurface orthogonal non-null Killing vector field are conformally flat. A similar theorem was proven for TMG in [23] in a coordinate-independent way and in [24] with explicit coordinates. Here we provide our proof with both methods. Hence to get nonconformally flat solutions, one must introduce twist or rotation. In addition, we briefly study all solutions of TMG that also solve EMG and give an explicit example which is the squashed $\mathrm{AdS}_{3}$ metric.

The outline of the paper is as follows: In Secs. II and III we study the causality of the theory in flat and AdS 
spacetimes, respectively, using the Shapiro time-delay computations. In Sec. IV we show that all the spacetimes that possess a hypersurface orthogonal Killing vector are conformally flat. In Sec. V, we show that the solutions of TMG are inherited by EMG as long as the coupling parameters of the theories are related in a prescribed way.

\section{CAUSALITY IN EXOTIC MASSIVE GRAVITY}

Here we study the local causality issue in exotic massive gravity via the computation of the Shapiro time delay or advance. Time advance would yield a noncausal theory, while time delay would be consistent with a causal one (see [16] and [17] for more on this). For this purpose, let us consider the source-coupled field equations of EMG [10],

$$
G_{\mu \nu}+\frac{1}{\mu} C_{\mu \nu}-\frac{1}{m^{2}} H_{\mu \nu}+\frac{1}{m^{4}} L_{\mu \nu}=\Theta_{\mu \nu}(T),
$$

where $\Theta_{\mu \nu}(T)$ is a complicated "energy-momentum" tensor which is on shell covariantly conserved and is given explicitly as

$$
\begin{aligned}
\Theta_{\mu \nu}(T)= & \frac{\lambda}{\mu} \hat{T}_{\mu \nu}-\frac{\lambda}{m^{2}} \epsilon_{\mu}{ }^{\rho \sigma} \nabla_{\rho} \hat{T}_{\nu \sigma}+\frac{2 \lambda}{m^{4}} \epsilon_{\mu}{ }^{\rho \sigma} \epsilon_{\nu}{ }^{\lambda \tau} C_{\rho \lambda} \hat{T}_{\sigma \tau} \\
& -\frac{\lambda^{2}}{m^{4}} \epsilon_{\mu}{ }^{\rho \sigma} \epsilon_{\nu}{ }^{\lambda \tau} \hat{T}_{\rho \lambda} \hat{T}_{\sigma \tau} ;
\end{aligned}
$$

here $\hat{T}_{\mu \nu}=T_{\mu \nu}-\frac{1}{2} g_{\mu \nu} T$ and $T_{\mu \nu}$ is covariantly conserved. The parameter $\lambda$ appears as a coupling constant between the source and the geometry in a nontrivial, nonhomogeneous way as can be seen in the last term of (2). The tensors on the left-hand side of (1) are defined as

$$
\begin{aligned}
& C_{\mu \nu}=\epsilon_{\mu}{ }^{\rho \sigma} \nabla_{\rho} S_{\nu \sigma}, \quad H_{\mu \nu}=\epsilon_{\mu}{ }^{\rho \sigma} \nabla_{\rho} C_{\nu \sigma}, \\
& L_{\mu \nu}=\frac{1}{2} \epsilon_{\mu}{ }^{\rho \sigma} \epsilon_{\nu}{ }^{\lambda \tau} C_{\rho \lambda} C_{\sigma \tau},
\end{aligned}
$$

where $S_{\mu \nu}:=R_{\mu \nu}-\frac{1}{4} g_{\mu \nu} R$. More explicitly, one has

$H_{\mu \nu}=\square S^{\mu \nu}-\nabla^{\mu} \nabla^{\nu} S+g^{\mu \nu} S_{\alpha \beta}^{2}-3 S^{\mu}{ }_{\alpha} S^{\alpha \nu}$,

$L_{\mu \nu}=\frac{1}{2} g_{\mu \nu} C_{\rho \sigma} C^{\rho \sigma}-C_{\mu \rho} C^{\rho}{ }_{\nu}$.

The theory (1), around its flat vacuum, has two massive spin-2 excitations with different masses, given as

$$
m_{ \pm}=m\left( \pm \frac{m}{2 \mu}+\sqrt{1+\frac{m^{2}}{4 \mu^{2}}}\right)
$$

reflecting its parity noninvariant nature. In the $\mu \rightarrow \infty$ limit, the masses coalesce: $m_{ \pm}=m$ for both helicity +2 and -2 modes. We shall also study this parity-invariant version of the theory. To analyze the causality issue in this theory, let us consider the shockwave metric created by a massless point particle moving in a fixed direction, say the $x$-direction. The shockwave metric written in two null, one spatial coordinates is

$$
d s^{2}=-d u d v+H(u, y) d u^{2}+d y^{2},
$$

with the null coordinates defined as $u:=t-x$ and $v:=$ $t+x$ and $y$ is the transverse coordinate. Taking the momentum of the massless source particle to be in the $+x$ direction, one has $p^{\mu}=|p|\left(\delta_{0}^{\mu}+\delta_{x}^{\mu}\right)$. Figure 1 depicts the spacetime region near the source. The energymomentum tensor of such a source has only one nonzero component, given as $T_{u u}=|p| \delta(y) \delta(u)$. For the shockwave ansatz (6), the scalar curvature $R$ vanishes and the only nonvanishing components of the Ricci, Cotton, and $H$ tensors are

$$
\begin{aligned}
R_{u u} & =G_{u u}=-\frac{1}{2} \frac{\partial^{2}}{\partial y^{2}} H(u, y), \quad C_{u u}=\frac{1}{2} \frac{\partial^{3}}{\partial y^{3}} H(u, y), \\
H_{u u} & =-\frac{1}{2} \frac{\partial^{4}}{\partial y^{4}} H(u, y),
\end{aligned}
$$

while the $L$ tensor vanishes identically. Then the EMG field equations, for the shockwave metric (6), reduce to a single fourth-order differential equation

$$
\begin{gathered}
\left(-1+\frac{1}{\mu} \partial_{y}+\frac{1}{m^{2}} \partial_{y}^{2}\right) \partial_{y}^{2} H(u, y) \\
=2 \lambda|p| \delta(u)\left(\frac{\delta(y)}{\mu}+\frac{\delta^{\prime}(y)}{m^{2}}\right)
\end{gathered}
$$

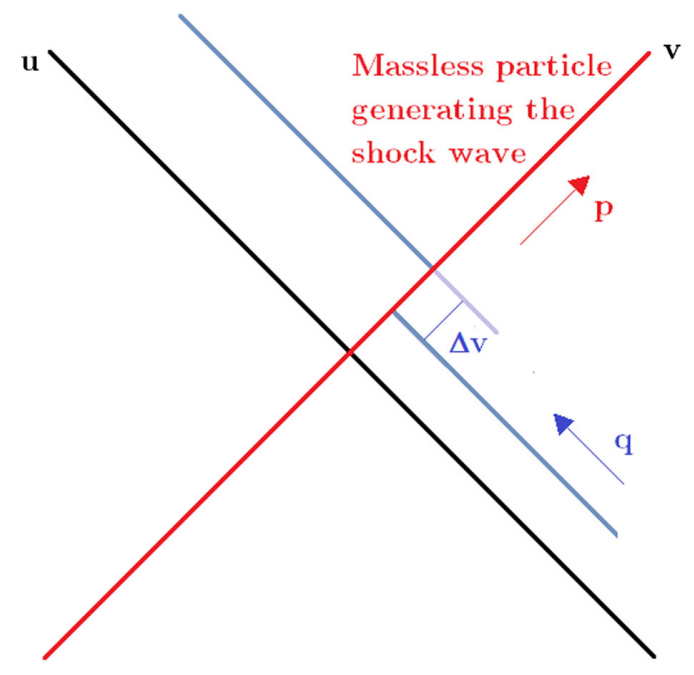

FIG. 1. Spacetime diagram depicting the shockwave geometry created by a massless particle with momentum $p$ moving in the $+x$ direction. Another massless test particle with momentum $q$ (blue line) propagating through this geometry experiences a time delay when it crosses the constant $u$ line. The time delay is denoted as a discontinuous jump $\Delta v$ in the null $v$ direction. Time advance would violate causality. 
where a prime denotes derivative with respect to the transverse coordinate $y$. The general solution of the last equation comes with four arbitrary functions $c_{1}, c_{2}, c_{3}$, and $c_{4}$ of the null coordinate $u$ and reads explicitly as

$$
\begin{aligned}
H(u, y)= & \lambda|p| \delta(u) \theta(y)\left\{\left(e^{m_{-} y}-1\right)\left(\frac{1}{m_{-}^{2}}+\frac{\mu+m_{+}}{\mu^{2}\left(m_{+}+m_{-}\right)}\right)\right. \\
& \left.+\frac{\left(e^{-m_{+} y}-1\right)}{m_{+}^{2}}\left(1-\frac{m^{2}}{\mu\left(m_{+}+m_{-}\right)}\right)+y\left(\frac{m_{-}-m_{+}}{m_{-} m_{+}}-\frac{4 \mu+m_{+}-m_{-}}{\left(2 \mu-m_{-}\right)\left(2 \mu+m_{+}\right)}\right)\right\} \\
& +\frac{1}{m^{4}}\left(m_{-}^{2} e^{-m_{+} y} c_{1}+m_{+}^{2} e^{m_{-} y} c_{2}\right)+c_{3}+c_{4} y .
\end{aligned}
$$

As noted, this is the most general solution, but one can fix the arbitrary functions by coordinate transformations in such a way that spacetime is asymptotically flat and given in a Cartesian form far away from the source. But this cannot be done with a single chart for the whole spacetime, so one can choose the $y>0$ part to be asymptotically flat in the Cartesian form. For more discussion on this issue, see [17,25]. A careful analysis leads to the following metric profile function with all arbitrary parameters fixed:

$$
\begin{aligned}
H(u, y)= & \frac{1}{m_{+}^{2}}\left(1-\frac{m^{2}}{\mu\left(m_{+}+m_{-}\right)}\right) e^{-m_{+} y} \lambda|p| \delta(u) \theta(y) \\
& -y|p| \delta(u)\left(\frac{m_{-}-m_{+}}{m_{-} m_{+}}-\frac{4 \mu+m_{+}-m_{-}}{\left(2 \mu-m_{-}\right)\left(2 \mu+m_{+}\right)}\right) \theta(-y) \\
& +\lambda|p| \delta(u)\left(\frac{1}{m_{-}^{2}}+\frac{\mu+m_{+}}{\mu^{2}\left(m_{+}+m_{-}\right)}+\frac{1}{m_{+}^{2}}-\frac{m^{2}}{\mu\left(m_{+}+m_{-}\right) m_{+}^{2}}\right) \theta(-y) \\
& -\lambda|p| \delta(u)\left(\frac{1}{m_{-}^{2}}+\frac{\mu+m_{+}}{\mu^{2}\left(m_{+}+m_{-}\right)}\right) e^{m_{-} y} \theta(-y) .
\end{aligned}
$$

Let us now consider a massless spinless test particle with momentum $q$ traversing the shockwave created by another massless spinless particle with momentum $p$, with an impact parameter $y=b>0$ as shown in the Fig. 1. In that region, (10) simplifies and the shockwave line element is given as

$$
\begin{aligned}
d s^{2}= & -d u\left(d v-\frac{1}{m_{+}^{2}}\left(1-\frac{m_{+}-m_{-}}{m_{+}+m_{-}}\right) e^{-m_{+} y} \lambda|p| \delta(u) d u\right) \\
& +d y^{2} .
\end{aligned}
$$

Clearly, as expected, the metric has a discontinuity in the null coordinate $u$ due to the distributional nature of the source. This discontinuity can be eliminated by redefining a new null coordinate $v_{\text {new }}$ at the impact parameter $b$ as

$$
v_{\text {new }}:=v+\frac{1}{m_{+}^{2}}\left(1-\frac{m_{+}-m_{-}}{m_{+}+m_{-}}\right) e^{-m_{+} b} \lambda|p| \theta(u),
$$

which gives rise to a time delay when the particle passes the $u=0$ line as can be explicitly seen from the equivalent expression:

$$
\Delta v=\frac{1}{m_{+}^{2}}\left(1-\frac{m}{\sqrt{m^{2}+4 \mu^{2}}}\right) e^{-m_{+} b} \lambda|p|
$$

Assuming $\lambda>0, \Delta v$ is positive for any value of the impact parameter. It is also important to note that, due to the parity-noninvariance of the theory, the test particle experiences a different time delay depending on whether it is moving in the $+x$ or $-x$ direction. For the impact parameter $y=b<0$, following the similar steps as above, one can find the corresponding time delay. So for causality, the only constraint is $\lambda>0$.

Furthermore, in the $\mu \rightarrow \infty$ limit and for the choice of $\lambda=m$, ${ }^{1}$ which corresponds to the parity-invariant version of EMG theory, the shift in the $v$ coordinate can be written as ${ }^{2}$

$$
\Delta v=\frac{1}{m} e^{-m b}|p|
$$

\footnotetext{
${ }^{1}$ For this choice and limit, we have the matter-coupled field equation [10]

$$
\begin{aligned}
G_{\mu \nu}-\frac{1}{m^{2}} H_{\mu \nu}+\frac{1}{m^{4}} L_{\mu \nu}= & -\frac{1}{m} \epsilon_{\mu}{ }^{\rho \sigma} \nabla_{\rho} \hat{T}_{\nu \sigma}+\frac{2}{m^{3}} \epsilon_{\mu}{ }^{\rho \sigma} \epsilon_{\nu}{ }^{\lambda \tau} C_{\rho \lambda} \hat{T}_{\sigma \tau} \\
& -\frac{1}{m^{2}} \epsilon_{\mu}{ }^{\rho \sigma} \epsilon_{\nu}{ }^{\lambda \tau} \hat{T}_{\rho \lambda} \hat{T}_{\sigma \tau} .
\end{aligned}
$$

Note that, in [10], a negative sign was erroneously forgotten in the first term of the source.

${ }^{2}$ For more details, see the Appendix. 
which is again positive for $m>0$. Note that in the opposite limit of $\mu \rightarrow 0$, the theory boils down to pure Cotton or Chern-Simons theory without a propagating degree of freedom and the metric is locally conformally flat with no interesting dynamics.

\section{A. Scalar field in a shockwave}

It pays to reproduce and extend the results of the above computation-done for null geodesics - to fields following [17]. In particular, the results become more transparent for a massless scalar field (still a test field with no backreaction) propagating in the background shockwave. For this purpose, let us consider the Klein-Gordon (KG) equation for a massless real scalar field,

$$
\square \phi=0,
$$

with $\square:=\nabla_{\mu} \nabla^{\mu}$; the equation reduces to a nontrivial partial differential equation

$$
\partial_{u} \partial_{v} \phi+H(u, y) \partial_{v}^{2} \phi-\frac{1}{4} \partial_{y}^{2} \phi=0,
$$

whose general solution seems elusive. But for our purposes, this is not needed: all we want is the approximate solution near the shockwave. In that case, the last term is negligible compared to the others and hence, the massless KG equation becomes

$$
\partial_{u} \partial_{v} \phi+H(u, y) \partial_{v}^{2} \phi=0,
$$

which is amenable to a $v$-integration. That integration brings a constant, which can be chosen to fit the boundary condition that in the $v \rightarrow \pm \infty$ limit, the scalar field vanishes. This reduces the equation to the following first order form:

$$
\partial_{u} \phi+H(u, y) \partial_{v} \phi=0,
$$

which admits a solution obtainable by the technique of separation of variables as $\phi(u, v, y):=U(u) V(v) Y(y)$. Then the solution with a momentum mode $p_{v}$ reads

$$
\phi(u, v, y)=Y(y) U\left(u_{0}\right) V(0) e^{i p_{v}\left(v-\int^{u} H\left(u^{\prime}, y\right) d u^{\prime}\right)} .
$$

From (20), it is clear that when a massless scalar particle/ field crosses the shockwave geometry with an impact parameter $b$, it picks up an Aharonov-Bohm type phase as

$$
\begin{aligned}
\phi\left(0^{+}, v, b\right) & =e^{-i p_{v} \int_{0^{-}}^{0^{+}} d u H(u, b)} \phi\left(0^{-}, v, b\right) \\
& =e^{-i p_{v} \Delta v} \phi\left(0^{-}, v, b\right) ;
\end{aligned}
$$

here $\Delta v$ is equivalent to the one given in (13) obtained via the geodesics computations. Next we extend the discussion to the anti-de Sitter spacetime.

\section{CAUSALITY IN ANTI-DE SITTER SPACE}

Let us consider the line element of $\mathrm{AdS}_{3}$ described in terms of Poincaré coordinates as

$$
d s^{2}=\frac{\ell^{2}}{y^{2}}\left(-2 d u d v+d y^{2}\right),
$$

where the null $u, v$ coordinates were defined in the previous section and take values in the whole real line while $y \in \mathbb{R}_{+}$. Once again, consider a massless source particle moving in the $+x$ direction in this background; then the resulting shockwave metric in the Brinkmann form can be taken as

$$
d s^{2}=\frac{\ell^{2}}{y^{2}}\left(-2 d u d v+F(u, y) d u^{2}+d y^{2}\right),
$$

with the profile function $F(u, y)$ to be determined below. The energy-momentum tensor of the point source with the prescribed motion described above at $y_{0}$ reads

$$
T_{u u}=|p| \frac{\ell}{y_{0}} \delta(u) \delta\left(y-y_{0}\right) .
$$

The source-coupled field equations of the parity-invariant version of EMG take the form ${ }^{3}$

$$
G_{\mu \nu}-\frac{1}{\ell^{2}} g_{\mu \nu}-\frac{1}{m^{2}} H_{\mu \nu}+\frac{1}{m^{4}} L_{\mu \nu}=\Theta_{\mu \nu}(T),
$$

and for the metric ansatz (23), they reduce to a single equation,

$$
\begin{aligned}
& \left(y^{3} \partial_{y}^{3}+2 y^{2} \partial_{y}^{2}+m^{2} \ell^{2}\left(-y \partial_{y}+1\right)\right) \partial_{y} F(u, y) \\
& \quad=2 m \ell^{2}|p| \delta(u)\left(\frac{y \delta\left(y-y_{0}\right)}{y_{0}}+\frac{y^{2} \delta^{\prime}\left(y-y_{0}\right)}{y_{0}}\right),
\end{aligned}
$$

whose solution is

$$
\begin{aligned}
F(u, y)= & \frac{\ell^{2} m \delta(u)|p|}{\ell^{2} m^{2}-1}\left(\left(\frac{y}{y_{0}}\right)^{-\ell m+1}+\left(\frac{y}{y_{0}}\right)^{\ell m+1}-\left(\frac{y}{y_{0}}\right)^{2}-1\right) \theta\left(y-y_{0}\right) \\
& +\frac{\ell^{2} m \delta(u)|p|}{\ell^{2} m^{2}-1}\left(c_{1}\left(\frac{y}{y_{0}}\right)^{-\ell m+1}+c_{2}\left(\frac{y}{y_{0}}\right)^{\ell m+1}+c_{3}\left(\frac{y}{y_{0}}\right)^{2}+c_{4}\right),
\end{aligned}
$$

\footnotetext{
${ }^{3}$ One can consider the generalized version by keeping the Cotton tensor, but the resulting equations are cumbersome without changing the ensuing discussion in a significant way.
} 
where all $c_{i}$ 's depend on $u$. They can be fixed by imposing appropriate boundary conditions and we shall do so after the following discussion. First let us check the flat space limit of the solution.

\section{A. The flat spacetime limit}

In the flat space limit $(\ell \rightarrow \infty)$, the solution (27) smoothly reduces to the flat space version which we have reproduced in the Appendix for the sake of completeness. To take the limit, let us introduce a new coordinate [17],

$$
y:=\ell e^{z / \ell},
$$

in which the $\mathrm{AdS}_{3}$ metric reads

$$
d s^{2}=-2 e^{-2 z / \ell} d u d v+d z^{2} .
$$

The flat and AdS shockwave profile functions are related as

$$
e^{-2 z / \ell} F(u, z)=H(u, z) \text {. }
$$

Consequently, in the $\ell \rightarrow \infty$ limit, one obtains

$$
\begin{aligned}
H(u, z)= & \frac{\delta(u)|p|}{m}\left(e^{-m\left(z-z_{0}\right)}+e^{m\left(z-z_{0}\right)}-2\right) \theta\left(z-z_{0}\right) \\
& +\frac{\delta(u)|p|}{m}\left(c_{1} e^{-m\left(z-z_{0}\right)}+c_{2} e^{m\left(z-z_{0}\right)}+c_{3}+c_{4}\right),
\end{aligned}
$$

which is the same result as the one found in the flat space analysis.

\section{B. Brown-Henneaux conditions on the $\mathrm{AdS}_{3}$ shockwave}

Imposing the Brown-Henneaux (BH) $\mathrm{AdS}_{3}$ boundary conditions [26] on (27), we can fix the arbitrary functions. $\mathrm{BH}$ boundary conditions prescribe decay conditions (as one approaches the connected boundary $y \rightarrow 0$ ) for the linearized metric perturbations $h_{\mu \nu}=g_{\mu \nu}-g_{\mu \nu}^{\mathrm{AdS}}$ as

$h_{u u} \simeq h_{u v} \simeq h_{v v} \simeq h_{y y} \simeq \mathcal{O}\left(y^{0}\right), \quad h_{u y} \simeq h_{v y} \simeq \mathcal{O}(y)$.

So one demands $F(u, y) \sim \mathcal{O}\left(y^{2}\right)$. The discussion bifurcates depending on the sign of $1-\ell m$; for the sake of concreteness, let us assume $m>1 / \ell$. Then it is clear to see that one must set $c_{1}=0$. Recall that, as one moves to $y \rightarrow \infty$, one approaches the disconnected point boundary of $\mathrm{AdS}_{3}$, we can choose $c_{2}=-1$ and $c_{3}=c_{4}=1$ to approach $\mathrm{AdS}_{3}$ on that boundary. Finally, the gauge-fixed shockwave solution reads

$$
\begin{aligned}
F(u, y)= & \frac{\ell^{2} m \delta(u)|p|}{\ell^{2} m^{2}-1}\left(\frac{y}{y_{0}}\right)^{1-\ell m} \theta\left(y-y_{0}\right) \\
& +\frac{\ell^{2} m \delta(u)|p|}{\ell^{2} m^{2}-1}\left(-\left(\frac{y}{y_{0}}\right)^{1+\ell m}+\left(\frac{y}{y_{0}}\right)^{2}+1\right) \\
& \times \theta\left(y_{0}-y\right) .
\end{aligned}
$$

We can now consider the Shapiro time-delay computation for a massless scalar field in the AdS shockwave geometry. In complete analogy with the flat space, one arrives at an Aharonov-Bohm phase and a time-delay given as

$$
\Delta v=\int_{0^{-}}^{0^{+}} d u F(u, y)
$$

Plugging (33) into this integral and going to the $z$-coordinates, for $z>z_{0}$, the time shift can be found to be

$$
\Delta v=\frac{|p| m}{m_{g}^{2}} e^{-\left(z-z_{0}\right)\left(m-\frac{1}{t}\right)},
$$

where $m_{g}$ is the graviton mass given as $m_{g}^{2}=m^{2}-1 / \ell^{2}$. Observe that, as was shown in the flat space analysis, Shapiro time delay is positive and so causality is not violated in EMG. Note that, if we take the $\ell \rightarrow \infty$ limit, we recover the flat space result (15) as expected.

\section{BIRKHOFF-LIKE THEOREM IN EXOTIC MASSIVE GRAVITY}

For spacetime dimensions $n>2+1$, the group of spherical symmetry $S O(n-1)$ is non-Abelian. This has a nontrivial consequence on spherically symmetric spacetimes. For example, in four-dimensional general relativity, $S O(3)$ symmetry with three Killing vector fields necessitates a fourth Killing vector field and, in particular, Ricciflat spherically symmetric metrics are static, which is the essence of the Birkhoff theorem (or more properly the Jebsen-Birkhoff theorem [27]). On the other hand, in $n=$ $2+1$ spacetime dimensions, which is our case here, the group of "spherical symmetry" is $S O(2)$ with a single Killing vector field. This symmetry does not rule out rotations unlike the higher-dimensional cases; namely, in the $(t, r, \phi)$ coordinates, $g_{t \phi}$ terms need not be zero. So the discussion of the 2+1-dimensional Birkhoff theorem needs more refinement compared to the four-dimensional case. Nevertheless, in topologically massive gravity, a nice theorem was established in a coordinate-independent way in [23] and in local coordinates in [24]. The essence of the theorem is as follows: in TMG without a cosmological constant, assuming a hypersurface orthogonal Killing vector field, all solutions are locally flat. Here, we extend this theorem to the EMG. Unlike the case in TMG, the scalar curvature is not constant and there are higher 
curvature terms in the equation, so the proof of the analogous theorem is more complicated.

Theorem: Any $2+1$-dimensional spacetime with the cylinder topology $\left(\Sigma_{2} \times S^{1}\right)$ having a non-null hypersurface orthogonal Killing vector field is conformally flat in exotic massive gravity.

Proof.-First let us show this with an explicit construction in local coordinates and later provide the coordinatefree version which is somewhat more involved. Assume local light-cone coordinates $(u, v, \phi)$ and take the hypersurface orthogonal Killing vector field to be $\xi=\partial_{\phi}$; then the metric, under the assumptions, can be taken as

$$
d s^{2}=-f(u, v) d u d v+g(u, v)^{2} d \phi^{2}
$$

here $f(u, v) g(u, v) \geq 0$ is assumed to keep the signature intact. Clearly $\xi$ satisfies the Killing property,

$$
\nabla_{\mu} \xi_{\nu}+\nabla_{\nu} \xi_{\mu}=0
$$

and it is also easy to show that it satisfies the hypersurface orthogonality,

$$
\xi_{\mu} \nabla_{\nu} \xi_{\sigma}+\xi_{\nu} \nabla_{\sigma} \xi_{\mu}+\xi_{\sigma} \nabla_{\mu} \xi_{\nu}=0
$$

The field equations of the theory in vacuum are

$$
\begin{aligned}
E_{\mu \nu} & :=G_{\mu \nu}+\Lambda g_{\mu \nu}+\frac{1}{\mu} C_{\mu \nu}-\frac{1}{m^{2}} H_{\mu \nu}+\frac{1}{m^{4}} L_{\mu \nu} \\
& =\mathcal{E}_{\mu \nu}+\frac{1}{\mu} C_{\mu \nu}=0 .
\end{aligned}
$$

In the second line we defined

$$
\mathcal{E}_{\mu \nu}:=G_{\mu \nu}+\Lambda g_{\mu \nu}-\frac{1}{m^{2}} H_{\mu \nu}+\frac{1}{m^{4}} L_{\mu \nu},
$$

since the crux of the argument is to show that the Cotton tensor will be orthogonal to this tensor. Observe also that we have included a cosmological constant. For the metric (36), one can compute $\mathcal{E}^{\mu}{ }_{\nu}$ and $C^{\mu}{ }_{\nu}$ and depict the nonzero parts as ${ }^{4}$

$$
\mathcal{E}^{\mu}{ }_{\nu}=\left(\begin{array}{ccc}
X_{1} & X_{2} & 0 \\
X_{3} & X_{4} & 0 \\
0 & 0 & X_{5}
\end{array}\right),
$$

and

\footnotetext{
${ }^{4}$ It is advantageous to study the $(1,1)$ tensor form of the field equations instead of the $(0,2)$ tensor form.
}

$$
C^{\mu}{ }_{\nu}=\left(\begin{array}{ccc}
0 & 0 & Y_{1} \\
0 & 0 & Y_{2} \\
Y_{3} & Y_{4} & 0
\end{array}\right),
$$

where $X_{i}$ and $Y_{i}$ are complicated functions of $f(u, v)$ and $g(u, v)$ and their derivatives which we shall not write here explicitly. The crucial observation is that the matrices $\mathcal{E}^{\mu}{ }_{\nu}$ and $C^{\mu}{ }_{\nu}$ are orthogonal to each other and hence, assuming the field equations (39), they must separately vanish. Vanishing of the Cotton tensor is the necessary and sufficient condition for a 3D metric to be conformally flat. Therefore, the theorem follows. To obtain conformally nonflat solutions, one must introduce twist, or the Killing vector field should not be hypersurface orthogonal.

We can verify the above result in a coordinate-free way following the computation in the TMG case given in [23]. The hypersurface orthogonal Killing vector field defines a parallel direction and two perpendicular directions which yield a natural splitting of the field equations. For this purpose, let us define the orthogonal projector $\perp$ as

$$
\perp_{\nu}^{\mu}:=\delta_{\nu}^{\mu}-\frac{\xi^{\mu} \xi_{\nu}}{\xi^{2}},
$$

where $\xi^{2}:=g_{\mu \nu} \xi^{\mu} \xi^{\nu} \neq 0$. So clearly $\perp_{\nu}^{\mu} \xi^{\nu}=0$. In what follows we shall denote the component of a tensor in the direction parallel to $\xi$ as $T_{\xi}$ and perpendicular to $\xi$ as $T_{\perp}$. Let us first show the following:

$$
C_{\xi}^{\xi}=0, \quad \mathcal{E}_{\xi}^{\xi} \neq 0,
$$

which are the bottom far right corners in (42) and (41). By definition

$$
\begin{aligned}
C_{\xi}^{\xi} & =C_{\nu}^{\mu} \xi_{\mu} \xi^{\nu}=\xi_{\mu} \xi^{\nu} \eta^{\mu \alpha \beta} \nabla_{\alpha}\left(R_{\beta \nu}-\frac{1}{4} g_{\beta \nu} R\right) \\
& =\xi_{\mu} \xi^{\nu} \eta^{\mu \alpha \beta} \nabla_{\alpha} R_{\beta \nu},
\end{aligned}
$$

where the scalar curvature term $R$ is dropped due to symmetry, not due to $R$ being a constant as in TMG. In fact $R$ is not assumed to be a constant. Pulling out the covariant derivative, one has

$$
C_{\xi}^{\xi}=\nabla_{\alpha}\left(\xi_{\mu} \xi^{\nu} \eta^{\mu \alpha \beta} R_{\beta \nu}\right)-\eta^{\mu \alpha \beta} R_{\beta \nu}\left(\xi_{\mu} \nabla_{\alpha} \xi^{\nu}+\xi^{\nu} \nabla_{\alpha} \xi_{\mu}\right) .
$$

To proceed we need some identities for the assumed $\xi$ derived in [23]. By taking the derivative $\nabla^{\nu}$ of (38), one finds the following identity:

$$
\xi_{\mu} R_{\alpha \nu} \xi^{\nu}=\xi_{\alpha} R_{\mu \nu} \xi^{\nu}
$$

which basically says that $\xi$ can be used to barter an index of the once-contracted Ricci tensor. This identity kills the first 
term in (46). For the second part we need the following identity which can be obtained by contracting (38) with $\xi^{\mu}$ :

$$
\nabla_{\mu} \xi_{\nu}=\frac{1}{2}\left(\xi_{\nu} \partial_{\mu} \log \left|\xi^{2}\right|-\xi_{\mu} \partial_{\nu} \log \left|\xi^{2}\right|\right) .
$$

Making use of this identity in the second part of (46), one has

$\eta^{\mu \alpha \beta} R_{\beta \nu}\left(\xi_{\mu} \nabla_{\alpha} \xi^{\nu}+\xi^{\nu} \nabla_{\alpha} \xi_{\mu}\right)=\frac{3}{2} \eta^{\mu \alpha \beta} R_{\beta}{ }^{\nu} \xi_{\mu} \xi_{\nu} \partial_{\alpha} \log \left|\xi^{2}\right|$,

which vanishes upon use of (47). Hence for a non-null hypersurface orthogonal Killing vector, one has $C_{\xi}^{\xi} \equiv 0$. On the other hand, one has

$\mathcal{E}_{\xi}^{\xi}=R^{\mu}{ }_{\nu} \xi_{\mu} \xi^{\nu}+\left(\Lambda-\frac{R}{2}\right) \xi^{2}-\frac{1}{m^{2}} H^{\mu}{ }_{\nu} \xi_{\mu} \xi^{\nu}+\frac{1}{m^{4}} L^{\mu}{ }_{\nu} \xi_{\mu} \xi^{\nu}$.

It is not difficult to see that there is no reason for this expression to vanish identically; for example, one has $R_{\xi}^{\xi}=-\xi^{\mu} \square \xi_{\mu} \neq 0$. Therefore (44) is proven.

Let us now prove the following:

$$
\mathcal{E}_{\perp}^{\xi} \equiv 0, \quad C_{\perp}^{\xi} \neq 0 .
$$

We have, by definition,

$$
\mathcal{E}^{\xi}{ }_{\perp}=R^{\mu}{ }_{\nu} \xi_{\mu} \perp_{\alpha}^{\nu}-\frac{1}{m^{2}} H^{\mu}{ }_{\nu} \xi_{\mu} \perp_{\alpha}^{\nu}+\frac{1}{m^{4}} L^{\mu}{ }_{\nu} \xi_{\mu} \perp_{\alpha}^{\nu} .
$$

Let us study this term by term as each term must vanish independently if the expression is expected to vanish identically due to the inhomogeneity of the expression in the mass parameter $m$. The first term is easy:

$$
\begin{aligned}
R_{\perp}^{\xi_{\perp}} & :=R^{\mu}{ }_{\nu} \xi_{\mu} \perp_{\alpha}^{\nu}=R_{\nu}^{\mu}{ }_{\nu} \xi_{\mu}\left(\delta_{\alpha}^{\nu}-\frac{\xi^{\nu} \xi_{\alpha}}{\xi^{2}}\right) \\
& =R^{\mu}{ }_{\alpha} \xi_{\mu}-R^{\mu} \nu \xi_{\mu} \frac{\xi^{\nu} \xi_{\alpha}}{\xi^{2}}=0,
\end{aligned}
$$

where we used the index-bartering identity (47) in the second term. Similarly the second term in (52) reads

$$
\begin{aligned}
H_{\perp}^{\xi} & :=H^{\mu}{ }_{\nu} \xi_{\mu} \perp_{\alpha}^{\nu} \\
& =\frac{1}{2} \eta^{\nu \mu}{ }_{\alpha} \nabla_{\nu}\left(\xi^{\sigma} C_{\mu \sigma}\right)-\frac{1}{2} \eta^{\nu \mu \lambda} \frac{\xi_{\lambda} \xi^{\alpha}}{\xi^{2}} \nabla_{\nu}\left(\xi^{\sigma} C_{\mu \sigma}\right),
\end{aligned}
$$

where we used the fact that $\xi$ is a Killing vector, yielding

$$
\mathcal{L}_{\xi} C_{\mu \nu}=0,
$$

which can be used to show the following relation:

$$
\xi_{\sigma} H_{\sigma}^{\lambda}=\frac{1}{2} \eta^{\nu \mu \lambda} \nabla_{\nu}\left(\xi^{\sigma} C_{\mu \sigma}\right) .
$$

It is clear that the $\alpha$ index in (54) must be $\perp$; hence one has

$$
H_{\perp}^{\xi}=\frac{1}{2} \eta^{\xi \perp} \nabla_{\xi} C^{\xi} .
$$

To show that this vanishes, we need to follow $\nabla_{\mu} C^{\mu}{ }_{\nu}=0$, which yields $\nabla_{\xi} C^{\xi} \perp=0$ since $C^{\perp}{ }_{\perp}=0$. Let us show in fact that $C^{\perp}{ }_{\perp}=0$ even when the scalar curvature is not constant:

$$
\begin{aligned}
C^{\perp}{ }_{\perp}:= & C^{\mu}{ }_{\nu} \perp_{\alpha}^{\nu} \perp_{\mu}^{\beta}{ }_{\mu} \\
= & \frac{1}{\xi^{2}}\left(\eta^{\beta \sigma \rho} \nabla_{\sigma} R_{\rho \alpha} \xi^{\mu} \xi_{\mu}-\eta^{\mu \sigma \rho} \nabla_{\sigma} R_{\rho \alpha} \xi^{\beta} \xi_{\mu}\right. \\
& \left.-\eta^{\beta \sigma \rho} \nabla_{\sigma} R_{\rho \nu} \xi^{\nu} \xi_{\alpha}\right) \\
& +\frac{\xi_{\mu} \nabla_{\sigma} R}{4 \xi^{2}}\left(-\eta^{\beta \sigma} \alpha \xi^{\mu}+\eta^{\mu \sigma}{ }_{\alpha} \xi^{\beta}+\eta^{\beta \sigma \mu} \xi_{\alpha}\right) .
\end{aligned}
$$

To see that this vanishes requires a couple of steps and the use of the three-dimensional identity

$$
\eta^{\lambda \nu \alpha} \xi^{\rho}=g^{\lambda \rho} \eta^{\beta \nu \alpha} \xi_{\beta}+g^{\nu \rho} \eta^{\lambda \beta \alpha} \xi_{\beta}+g^{\alpha \rho} \eta^{\lambda \nu \beta} \xi_{\beta},
$$

together with the Killing property $\mathcal{L}_{\xi} R_{\mu \nu}=0$ and (47). After making use of these, one can show that the first and second lines of (58) vanish identically separately.

Similarly the third term in (52) reads

$$
\begin{aligned}
L_{\perp}^{\xi} & :=L^{\mu}{ }_{\nu} \xi_{\mu} \perp_{\alpha}^{\nu}=\left(\frac{1}{2} \delta_{\nu}^{\mu} C_{\rho \sigma}^{2}-C^{\mu}{ }_{\sigma} C^{\sigma}{ }_{\nu}\right) \xi_{\mu} \perp_{\alpha}^{\nu} \\
& =-C^{\mu}{ }_{\sigma} C^{\sigma}{ }_{\nu} \xi_{\mu} \perp_{\alpha}^{\nu} \\
& =-C^{\xi}{ }_{\perp} C^{\perp}{ }_{\xi} \perp_{\alpha}^{\xi}=0,
\end{aligned}
$$

where we used the fact that $C_{\xi}^{\xi}=0$ and $C^{\perp}{ }_{\perp}=0$. One can also show that $\mathcal{E}^{\perp}{ }_{\perp} \neq 0$; hence the theorem follows and one must introduce twist to find conformally nonflat solutions.

\section{ALL SOLUTIONS OF TMG SOLVING EXOTIC MASSIVE GRAVITY}

Field equations of EMG are highly complicated, but it is clear that all Einstein metrics solve these equations. To move beyond Einstein metrics, let us consider all solutions of TMG that solve EMG. As the solutions of TMG are compiled in a nice paper [28], we shall not go into an extended discussion here, but just find the conditions that are needed to carry the TMG to solutions to the current theory. Let us assume that the metric $g_{\mu \nu}$ solves the TMG whose topological mass is $1 / a$; hence it satisfies the following equations:

$$
C_{\mu \nu}=a \tilde{R}_{\mu \nu}, \quad R=k,
$$


where $a$ and $k$ are constant and $\tilde{R}$ is the traceless Ricci tensor defined as $\tilde{R}_{\mu \nu}=R_{\mu \nu}-\frac{1}{3} g_{\mu \nu} R$. To search for solutions of (39), we assume that (61) also holds. Therefore, we are searching for constant scalar curvature solutions. The trace part of the field equations (39) is

$$
-\frac{R}{2}+3 \Lambda+\frac{1}{2 m^{4}} C_{\mu \nu} C^{\mu \nu}=0,
$$

and the traceless part is

$$
\tilde{R}_{\mu \nu}+\frac{1}{\mu} C_{\mu \nu}-\frac{1}{m^{2}} H_{\mu \nu}+\frac{1}{m^{4}} \tilde{L}_{\mu \nu}=0,
$$

where $\tilde{L}_{\mu \nu}=\frac{1}{3} g_{\mu \nu} C_{\rho \sigma}^{2}-C_{\mu \rho} C_{\nu}^{\rho}$. Let us define the following curvature invariants:

$$
I=\tilde{R}_{\nu}^{\mu} \tilde{R}_{\mu}^{\nu}, \quad J=\tilde{R}_{\alpha}^{\mu} \tilde{R}_{\beta}^{\alpha} \tilde{R}_{\mu}^{\beta},
$$

which are relevant to the classification of the solutions (see $[28,29]$ for more on this). Contracting (63) with $\tilde{R}_{\mu \nu}$ and making use of the TMG equation, one arrives at

$$
I\left(1+\frac{a}{\mu}-\frac{a^{2}}{m^{2}}\right)-\frac{a^{2}}{m^{4}} J=0,
$$

where $I=\frac{2 m^{4}}{a^{2}}\left(\frac{R}{2}-3 \Lambda\right)$, which comes from (62). Then plugging this into the previous equation, one has

$$
J=\frac{2 m^{8}}{a^{4}}\left(1+\frac{a}{\mu}-\frac{a^{2}}{m^{2}}\right)\left(\frac{R}{2}-3 \Lambda\right) .
$$

So the solutions of TMG also solve EMG as long as these $I$ and $J$ equations are satisfied. Let us give an explicit example, which is called the timelike squashed $\mathrm{AdS}_{3}$,

$d s^{2}=\frac{\lambda^{2}-4}{2 R}\left(-\lambda^{2}(d \tau+\cosh \theta d \phi)^{2}+d \theta^{2}+\sinh ^{2} \theta d \phi^{2}\right)$,

with the squashing parameter $\lambda$ (not to be confused with the coupling constant of the earlier sections), and the constant scalar curvature of this metric is $R$. The metric (67) is a solution to EMG if $\lambda$ and $R$ have real solutions in terms of $\mu, \Lambda$, and $m$ as given in the following equations:

$\mu=\frac{\sqrt{18 R}\left(\lambda^{2}-4\right)^{3 / 2} m^{4} \lambda}{2\left(\lambda^{2}-4\right)^{2} m^{4}-9 \lambda^{2}\left(\lambda^{2}-4\right) m^{2} R+12 \lambda^{2}\left(\lambda^{2}-1\right) R^{2}}$,

$\Lambda=\frac{R\left(\left(\lambda^{2}-4\right)^{3} m^{4}-24 \lambda^{2}\left(\lambda^{2}-1\right)^{2} R^{2}\right)}{6\left(\lambda^{2}-4\right)^{3} m^{4}}$.

To search for solutions which are more general than the ones that solve TMG, one can resort to the method developed in [30].

\section{CONCLUSIONS}

In [16], rather unexpectedly, the Einstein-Gauss-Bonnet theory was shown to violate causality for any sign of the Gauss-Bonnet coupling constant. That seems to be a major blow for effective gravity theories. But luckily, string theory with an infinite tower of intermediate states can solve the problem [19]. Interestingly, despite having their own problems, various three-dimensional massive gravity theories were shown to not suffer from the causality violations, since a detailed study shows that the conditions coming from causality are not in conflict with the ones coming from unitarity. These theories were discussed in [17] save the recently constructed EMG theory which has not been hitherto studied along these lines. Here we discussed the issue of local causality in EMG in asymptotically flat and AdS spacetimes using the Shapiro timedelay computation for massless test particles and scalar fields in a shockwave geometry created by a massless source. Despite having a ghost, there is a time delay for any impact parameter between the source and the test field instead of a time advance; hence causality is not violated. In addition, we have studied some exact solutions in the theory and proved that all solutions with a hypersurface orthogonal Killing vector field are conformally flat. To go beyond conformally flat solutions, rotation must be introduced.

\section{APPENDIX: SOME DETAILS OF CAUSALITY IN THE PARITY-INVARIANT VERSION OF EMG}

The field equations of parity-invariant version of EMG are [10]

$$
G_{\mu \nu}-\frac{1}{m^{2}} H_{\mu \nu}+\frac{1}{m^{4}} L_{\mu \nu}=\Theta_{\mu \nu}(T),
$$

where $\Theta_{\mu \nu}(T)$ is the energy-momentum tensor and it is given as

$$
\begin{aligned}
\Theta_{\mu \nu}(T)= & -\frac{1}{m} \epsilon_{\mu}{ }^{\rho \sigma} \nabla_{\rho} \hat{T}_{\nu \sigma}+\frac{2}{m^{3}} \epsilon_{\mu}{ }^{\rho \sigma} \epsilon_{\nu}{ }^{\lambda \tau} C_{\rho \lambda} \hat{T}_{\sigma \tau} \\
& -\frac{1}{m^{2}} \epsilon_{\mu}{ }^{\rho \sigma} \epsilon_{\nu}{ }^{\lambda \tau} \hat{T}_{\rho \lambda} \hat{T}_{\sigma \tau} ;
\end{aligned}
$$

here $\hat{T}_{\mu \nu}=T_{\mu \nu}-\frac{1}{2} g_{\mu \nu} T$. For the shockwave metric, field equations (A1) reduce to a single equation,

$$
\left(-1+\frac{1}{m^{2}} \partial_{y}^{2}\right) \partial_{y}{ }^{2} H(u, y)=2|p| \delta(y) \delta(u),
$$

whose general solution can be found to be

$$
\begin{aligned}
H(u, y)= & \frac{|p| \delta(u)}{m}\left(e^{-m y}+e^{m y}-2\right) \theta(y) \\
& +\frac{1}{m}\left(c_{1} e^{m y}-c_{2} e^{-m y}\right)+c_{3},
\end{aligned}
$$


with $c_{i}$ 's that depend on the null coordinate $u$. By gauge fixing as was done in the text for the more general theory, the solution takes the following form:

$H(u, y)=\frac{|p| \delta(u)}{m} e^{-m y} \theta(y)+\frac{|p| \delta(u)}{m}\left(-e^{m y}+2\right) \theta(-y)$.
Finally, using the discontinuity in this profile function, one can calculate the time delay of a signal passing at an impact parameter $b$ as

$$
\triangle v=\frac{|p|}{m} e^{-m|b|},
$$

which is positive and matches (15) for $m>0$.
[1] S. Deser, R. Jackiw, and S. Templeton, Three-Dimensional Massive Gauge Theories, Phys. Rev. Lett. 48, 975 (1982); Topologically massive gauge theories, Ann. Phys. (N.Y.) 140, 372 (1982).

[2] E. A. Bergshoeff, O. Hohm, and P. K. Townsend, Massive Gravity in Three Dimensions, Phys. Rev. Lett. 102, 201301 (2009).

[3] A. Sinha, On the new massive gravity and AdS/CFT, J. High Energy Phys. 06 (2010) 061.

[4] I. Gullu, T. C. Sisman, and B. Tekin, Born-Infeld extension of new massive gravity, Classical Quantum Gravity 27, 162001 (2010).

[5] E. Bergshoeff, O. Hohm, W. Merbis, A. J. Routh, and P. K. Townsend, Minimal Massive 3D Gravity, Classical Quantum Gravity 31, 145008 (2014).

[6] M. R. Setare, On the generalized minimal massive gravity, Nucl. Phys. B898, 259 (2015).

[7] B. Tekin, Bulk and boundary unitary gravity in $3 \mathrm{D}: \mathrm{MMG}_{2}$, Phys. Rev. D 92, 024008 (2015).

[8] E. Altas and B. Tekin, Exact solutions and the consistency of 3D minimal massive gravity, Phys. Rev. D 92, 025033 (2015).

[9] E. Altas and B. Tekin, Holographically viable extensions of topologically massive and minimal massive gravity, Phys. Rev. D 93, 025033 (2016).

[10] M. Ozkan, Y. Pang, and P. K. Townsend, Exotic massive 3D gravity, J. High Energy Phys. 08 (2018) 035.

[11] M. Chernicoff, G. Giribet, N. Grandi, and J. Oliva, Vacua of exotic massive 3D gravity, J. High Energy Phys. 08 (2018) 087.

[12] G. Alkac, M. Tek, and B. Tekin, Bachian gravity in three dimensions, Phys. Rev. D 98, 104021 (2018).

[13] R. B. Mann, J. Oliva, and S. N. Sajadi, Energy of asymptotically AdS black holes in exotic massive gravity and its log-extension, J. High Energy Phys. 05 (2019) 131.

[14] G. Giribet and J. Oliva, More on vacua of exotic massive 3D gravity, Phys. Rev. D 99, 064021 (2019).

[15] G. Alkac, L. Basanisi, E. Kilicarslan, and B. Tekin, Unitarity problems in $3 D$ gravity theories, Phys. Rev. D 96, 024010 (2017).

[16] X. O. Camanho, J. D. Edelstein, J. Maldacena, and A. Zhiboedov, Causality constraints on corrections to the graviton three-point coupling, J. High Energy Phys. 02 (2016) 020.

[17] J. D. Edelstein, G. Giribet, C. Gomez, E. Kilicarslan, M. Leoni, and B. Tekin, Causality in 3D massive gravity theories, Phys. Rev. D 95, 104016 (2017).

[18] I. I. Shapiro, Fourth Test of General Relativity, Phys. Rev. Lett. 13, 789 (1964).

[19] G. D’Appollonio, P. Di Vecchia, R. Russo, and G. Veneziano, Regge behavior saves String Theory from causality violations, J. High Energy Phys. 05 (2015) 144.

[20] P. C. Aichelburg and R. U. Sexl, On the gravitational field of a massless particle, Gen. Relativ. Gravit. 2, 303 (1971).

[21] T. Dray and G. 't Hooft, The gravitational shock wave of a massless particle, Nucl. Phys. B253, 173 (1985).

[22] M. Banados, C. Teitelboim, and J. Zanelli, The Black Hole in Three-Dimensional Space-Time, Phys. Rev. Lett. 69, 1849 (1992).

[23] A. N. Aliev and Y. Nutku, A theorem on topologically massive gravity, Classical Quantum Gravity 13, L29 (1996).

[24] M. Cavaglia, The Birkhoff theorem for topologically massive gravity, Gravitation Cosmol. 5, 101 (1999).

[25] S. Deser, J. G. McCarthy, and A. R. Steif, Ultra-Planck scattering in $D=3$ gravity theories, Nucl. Phys. B412, 305 (1994).

[26] J. D. Brown and M. Henneaux, Central charges in the canonical realization of asymptotic symmetries: An example from three-dimensional gravity, Commun. Math. Phys. 104, 207 (1986).

[27] S. Deser, Introduction to Jebsen's paper, Gen. Relativ. Gravit. 37, 2251 (2005).

[28] D. D. K. Chow, C. N. Pope, and E. Sezgin, Classification of solutions in topologically massive gravity, Classical Quantum Gravity 27, 105001 (2010).

[29] M. Gurses, T. C. Sisman, and B. Tekin, Some exact solutions of all $f\left(R_{\mu \nu}\right)$ theories in three dimensions, Phys. Rev. D 86, 024001 (2012).

[30] M. Gurses, Killing vector fields in three dimensions: A method to solve massive gravity field equations, Classical Quantum Gravity 27, 205018 (2010); Erratum, Classical Quantum Gravity 29, 059501(E) (2012). 